\title{
Rough Coat Brussels Griffon
}

National Cancer Institute

\section{Source}

National Cancer Institute. Rough Coat Brussels Griffon. NCI Thesaurus. Code C53926.

The Griffon is a sturdy toy dog of square proportions with a domed head, undershot jaw, and a very short nose. Rough-Coated variety has a harsh wiry and dense coat. Griffons come in red, a mixture of red-brown and black, black and tan, and solid black. Ears are generally cropped. Height: 7-8 inches (18-20 cm.) Weight: 6-12 pounds (2.5-5.5 kg.) 